\title{
OPTIMIZATION OF THE RIB STRUCTURE INSIDE COOLING CHANNELS WITH GENETIC ALGORITHMS
}

\author{
A. HAASENRITTER \& B. WEIGAND \\ Institute of Aerospace Thermodynamics, University of Stuttgart, Germany.
}

\section{ABSTRACT}

To increase the thermal efficiency of industrial gas turbines, the performance of the internal cooling channels is of utmost importance. These cooling channels are often equipped with ribs on the top and bottom walls to increase the turbulence and the mixing of the flow. Depending on the position of the cooling channel in the turbine blade and the external heat load, the ribs have to be designed differently. Until now, the decision regarding the optimal design parameters was mainly based on the knowledge of the engineer. The present work investigates genetic algorithms as an optimization method to find optimal designs for the rib configuration in internal cooling channels. The main optimization targets are the enhancement of the heat transfer with a low increase in pressure drop. Results are presented for simplified 2D as well as realistic 3D cooling channel configurations.

Keywords: cooling channels, genetic algorithms, leading edge channel, turbulence generators.

\section{INTRODUCTION}

In the present study, a numerical method is investigated for the optimization of the rib structure inside cooling channels. Cooling channels are used for the internal convective cooling of gas turbine blades. The channels are mostly designed as multi-pass systems through the blade. In the main part of the blade, these channels have square or rectangular cross sections, while in the leading and trailing edge region the channels have triangular cross sections. Such channels normally have roughness elements, for example ribs, along two walls. Due to the ribs, the turbulence and the mixing of the flow is enhanced. To increase the thermal efficiency, different rib designs are used, as can be seen in the wide range of rib angles $\alpha$, rib heights $e$ and inner pitch distances $p$ considered in many studies. A typical gas turbine blade with cooling channels is shown in Fig. 1. The present work investigates an optimization method based on genetic algorithms (GAs) to find optimal designs for the rib configuration in an internal cooling channel.

Until now, the choice of the rib angle, rib height and inner pitch distance was mainly based on the engineers' knowledge, obtained from experiments and sometimes numerical computations. Finding an adequate design is a time-consuming task due to the numerous parameters influencing the performance of a cooling channel. Even small changes can influence the turbulent flow and the heat transfer and pressure loss in the channel. One can find a large number of experimental investigations about the heat transfer performance and friction factor characteristics in cooling channels. Some of the important experimental works are summarized in [1-3]. Numerical calculations offer a cheap alternative to experimental investigations and have shown good results in previous studies that dealt with heat transfer in internal cooling channels $[4,5]$. In future, when turbulence models are further developed, they might have the potential to replace more and more experimental work.

GAs are an abstraction of natural phenomena. They try to simulate the mechanisms of natural selection or evolution copied from biological organisms. Due to the high complexity of the turbulent flow and heat transfer problems appearing in cooling channels, traditional search algorithms are often not applicable. Furthermore, as gradient-based methods, they often result in local minima or maxima. This problem cannot appear in GAs due to the random search procedure. The whole optimization process is based on the automatic coupling of a GA with other software tools (for the geometry generation ProEngineer [6], for the grid generation CENTAUR [7] and for the calculation of the 

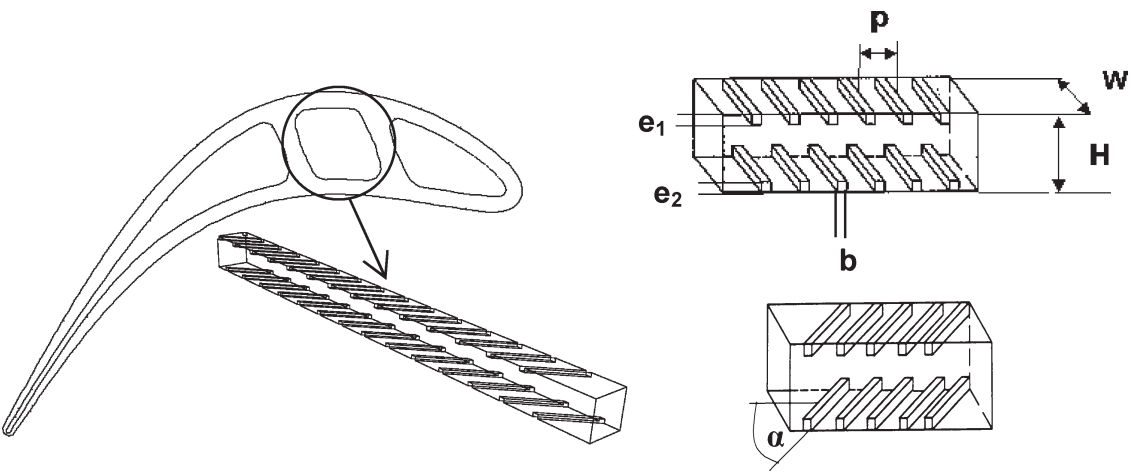

Figure 1: Turbine blade with internal cooling channel geometry.

flow field FLUENT [8]). The present paper deals with one very simple 2D geometry, which has been selected because of the reduced computing time. After the whole optimization process and all the parameters for the GAs are tested, we will switch to 3D optimization. In a cooling channel since the main optimization targets are the maximization of the Nusselt number and the minimization of the friction factor, different objective functions are investigated. It can be shown that the optimal rib configuration is highly dependent on the chosen objective function and that a better alternative to optimize the present problem can be offered by multi-objective genetic algorithms (MOGAs).

\section{BASIC PRINCIPLES OF GAS}

In the following section, the basic principles of single-objective genetic algorithms (SOGAs) and MOGAs will be explained. Both mimic the principles of natural genetics and natural selection and offer an alternative to traditional search methods to solve complex engineering optimization problems.

The main optimization target for both methods is to maximize or minimize a single $M=1$ (SOGA) or a set $M>1$ (MOGA) of objective functions $g$ :

$$
\begin{gathered}
\max / \min g_{m}(\boldsymbol{x}), \quad \text { with } m=1, \ldots, M, \\
a_{i}^{(\mathrm{L})} \leq x_{i} \leq b_{i}^{(\mathrm{U})} .
\end{gathered}
$$

The solution of the maximization problem is a $n$-dimensional vector $\boldsymbol{x}=\left(x_{1}, \ldots, x_{n}\right)$ in the search space, which is restricted for each variable through the lower boundaries $a_{i}^{(\mathrm{L})}$ and the higher boundaries $b_{i}^{(\mathrm{U})}$.

\subsection{Single-objective genetic algorithms}

For the SOGA, an optimization process starts with a random initial population of size $N$. Binary strings give the representation of the individuals. Thereafter, each string or chromosome is evaluated to find the corresponding fitness value. To create a new population of offspring, three operators have to be applied to the parent population: reproduction, crossover and mutation. This procedure is continued until a termination criterion is met. Each operation cycle including the evaluation of fitness is denoted as generation. A flowchart of the working principle of a GA is shown in Fig. 2. During the reproduction process, an improvement of the average performance of the individuals is achieved. The probability to generate offspring is higher for chromosomes with a better performance or higher 


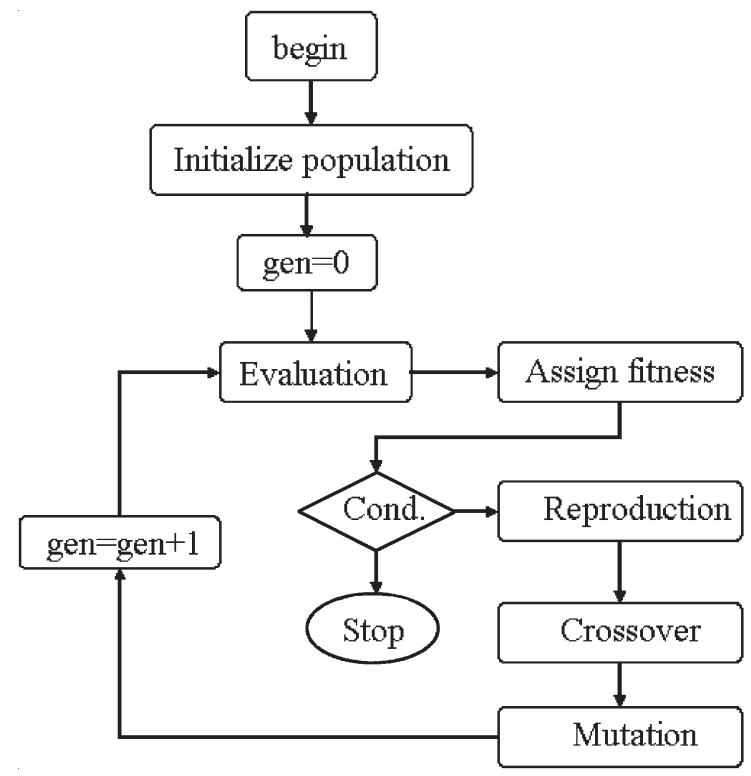

Figure 2: Working principle of a GA.

fitness value than for chromosomes with a low performance. A more detailed description of the GA used for the actual problem can be found in [9].

\subsubsection{Representation of individuals}

The decision variables $x_{i}$ are represented in binary strings. Let us assume that we have two decision variables, the normalized rib height $x_{1}=e / H$ and the normalized rib width $x_{2}=b / e$. To decode each of the two decision variables we use 4 bits, thereby making the overall length of the string equal to 8 . The length of the string is also a measure of the accuracy, since for 4 bits only 16 distinct values of a variable are possible to be obtained. The following string

$$
(0100)_{e / H}(0111)_{b / e}
$$

represents one possible individual representing one solution in the cooling channel. To decode these values, first, we have to calculate for each variable the value in binary arithmetic back to the decimal system:

$$
(0100)_{2}=(4)_{10} \text { and }(0111)_{2}=(7)_{10} .
$$

According to the allowed domain of the search space for the two variables:

$$
\begin{gathered}
0.05 \leq e / H \leq 0.3, \\
0.5 \leq b / e \leq 2,
\end{gathered}
$$

a linear mapping has to be applied to obtain the corresponding design variable:

$$
e / H=a_{i}+\left(b_{i}-a_{i}\right) /\left(2^{4}-1\right)=0.05+(0.3-0.05) /(15) \times 4=0.1166 .
$$


Thus, the decision variable (0100 0111) represents a sample channel with rib height $e / H=0.1166$ and rib width $b / e=1.2$.

\subsubsection{Fitness value}

The fitness value is a kind of measure for the performance of a single chromosome. This value is of high importance because only chromosomes with a performance that is above average have a high chance to reproduce attributes of themselves for the next generation. To evaluate the fitness of an individual, the original variables have to be obtained from the binary representation. The fitness function is in most cases equal to the objective function, which has to be maximized. In the case of a cooling channel, the objective function will contain the Nusselt number, which has to be maximized, and the reciprocal of the friction factor, which has to be minimized.

\subsubsection{Reproduction}

The primary objective of the reproduction operator is to duplicate good solutions in a population, while keeping the population size constant. This is achieved by identifying good and above-average solutions in a population by means of the fitness function, make multiple copies of them and eliminate bad solutions from the population so that multiple copies of good solutions can be placed in the population. There exist many reproduction operators, whereas in the present study the stochastic remainder selection is applied. It is a more stable version of the roulette wheel selection. Both reproduction methods are explained in detail in [9].

\subsubsection{Crossover and mutation}

Since reproduction does not produce any new solutions in the population, new individuals have to be created by crossover or mutation. In Fig. 3, examples of a crossover and a mutation are shown. The main principle of crossover is that two strings are picked from the mating pool at random and some portion of the strings is exchanged between them to create new strings. The crossover between two strings is performed with a probability given by the crossover probability $p_{\mathrm{c}}$. In a single-point crossover operation as used in the present program, this is performed by randomly choosing a cross site along the strings and by exchanging all the bits on the right side of the crossing site. To keep diversity in the population, the mutation operator changes a 1 to a 0 and vice versa depending on the predefined mutation probability $p_{\mathrm{m}}$.

CROSSOVER:

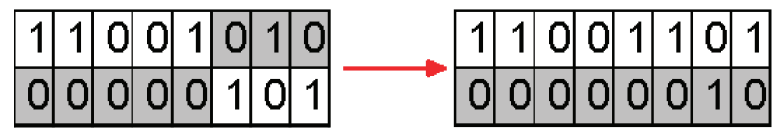

MUTATION:

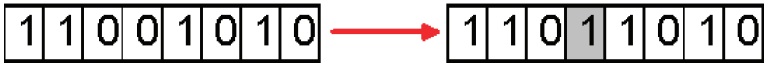

Figure 3: Crossover and mutation of binary chromosomes. 
2.2 Multi-objective genetic algorithms

In contrast to the SOGA, which optimizes a single objective function, the MOGA [10] optimizes multiple objective functions, which often have conflicting optimization targets. For this reason, it is no longer possible to find a single optimum for all different conflicting functions. Thus a new one, especially adapted to the multi-criterion problem, must replace the optimality concept used in scalar optimization. To compare two solutions with each other, the concept of domination has to be introduced. Through the application of the dominance principle, the set of optimal solutions can be found, which are building the Pareto-optimal front (POF) in the solution space. In the following section, some definitions about non-dominating sets and a POF will be given since they will be useful for understanding the MOGA.

For the following definitions, we assume that there are $M$ objective functions. The relation $i>j$ means that a solution $i$ is better than a solution $j$ independent of the kind of optimization problem. For a minimization problem, it means that the solution $i$ is smaller than $j$; for a maximization problem, it means that the solution $i$ is bigger than $j$. Similarly, $i<j$ means that the solution $i$ is worse than $j$.

\subsubsection{Definition 1}

A solution $x^{(1)}$ is said to dominate the other solution $x^{(2)}$, if both conditions 1 and 2 are true:

1. The solution $x^{(1)}$ is no worse than $x^{(2)}$ in all objectives, or $f_{j}\left(x^{(1)}\right)<f_{j}\left(x^{(2)}\right)$ for all $j=1, \ldots, M$.

2. The solution $x^{(1)}$ is strictly better than $x^{(2)}$ in at least one objective, or $f_{j}\left(x^{(1)}\right)>f_{j}\left(x^{(2)}\right)$ for at least one $j \in\{1, \ldots, M\}$.

The expression of a solution $x^{(1)}$ is said to dominate the other solution $x^{(2)}$; is equivalent to a solution $x^{(1)}$; and is non-dominated by the other solution $x^{(2)}$.

\subsubsection{Definition 2 (non-dominated set)}

Among a set of solutions $P$, the non-dominated set of solutions $P^{\prime}$ are those that are not dominated by any member of the set $P$. When the set $P$ is the entire search space, the resulting non-dominated set $P^{\prime}$ is called the Pareto-optimal set.

After the basic concepts are stated, the optimization algorithm used for multi-objective problems will be explained. In the present work, the 'Elitist non-dominated sorting algorithm' developed by Deb [10] will be applied. The working principle of a MOGA is basically the same as in a SOGA with the presentation of solutions as binary strings and reproduction mechanisms using crossover and mutation. The main difference can be found in the assignment of the fitness values. Let us assume an initial population $P_{0}$ of size $N$. First the whole set is subdivided into non-dominated sets and each solution gets a fitness value according to the value of the non-dominated set. Individuals in the best front get a 1, in the second front a 2 and so on. According to the SOGA, the offspring population $Q_{0}$ is obtained by reproduction, crossover and mutation. For the next generations, the following scheme is repeated.

The set $P_{t}$ of size $N$ is combined with the offspring generation $Q_{t}$ of size $N$ to obtain the new set $R_{t}$ with $2 N$ individuals. Next, the set $R_{t}$ will be subdivided in non-dominated fronts. To get the new population $P_{t+1}$ of size $N$ the best non-dominated front will be included, followed by the second front and so on until all positions in the new population are filled up. If there are more individuals in a non-dominated set than in places left in the new generation, an additional sorting algorithm has to be applied. The crowding distance sort assigns a second fitness value to the individuals, which is in relation to the distance of the two nearest neighbors. Solutions in sparsely populated regions have a 
higher chance to be accepted in the offspring generation. A detailed description about the algorithm can be found in [10]. Due to the non-dominated sorting of the parent and offspring population $R_{t}$, it is ensured that the elitists of a population are directly carried over to the next generation and cannot be replaced by their own offspring.

The two main optimization goals of a MOGA are to find a set of solutions as close as possible to the POF and place the individual along this front as diverse as possible to display the devolution as clearly as possible.

\section{RIB STRUCTURE OPTIMIZATION}

The aim of the present optimization is to find the optimal rib structure for internal cooling channels of gas turbine blades. The cooling channel geometry mostly has ribs on the bottom and top wall. The position and shape of these ribs along the two walls can be changed and they have to be optimized. The heat transfer rate along the walls in contact with the cooling fluid should be as high as possible and simultaneously the pressure loss has to be as low as possible. Of course, these optimization goals are only selected as examples. It is easily possible to change these goals.

Before the whole optimization process starts, the objective function, as a combination of the important magnitudes, the Nusselt number and the friction factor, has to be defined. This will be done in the next paragraph where the optimization results are presented.

To obtain the information needed for a given rib configuration, the whole flow and heat transfer problem has to be solved by numerical calculations. To solve the turbulent flow and heat transfer problem, four main steps have to be performed: geometry generation with the CAD tool ProE [6], grid generation with the unstructured hybrid grid generator CENTAUR [7], solving of the flow and energy equations for the turbulent flow with the CFD program FLUENT [8] and post-processing to obtain the characteristic values for the flow. The execution of all software tools and the handling of the interfaces are controlled by a script file. The whole process is running in the background; thus, no user interaction is needed. Figure 4 shows a flowchart of the interaction of the software tools with the GA.

The following points have to be considered in more detail for the program.

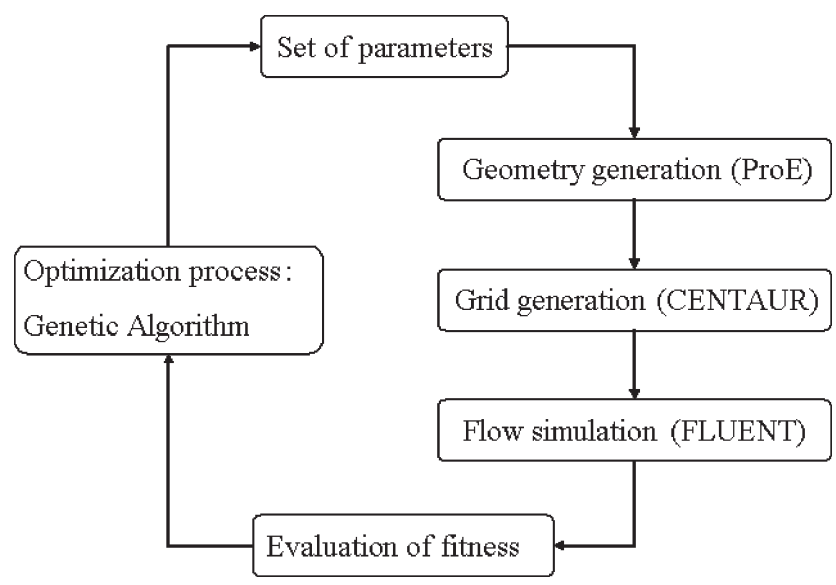

Figure 4: Flowchart showing the interaction of the software tools. 


\subsection{Geometry}

For the optimization procedure, the design parameters have to be defined. Typical parameters in an internal cooling channel are the heights $e$ and the widths $b$ of the ribs, the distance between the ribs $d$, the inner pitch distance $p$ and the rib angle $\alpha$. The domain boundaries for the different decision variables also have to be defined.

\subsection{ProE}

For the geometry generation, the CAD tool ProE [6] is used. A planar model with the new geometry definitions is generated by the use of trail files executing the same sequence of commands for every geometry. The change of the geometry definitions is given via relationships, which can be adopted for every case. The geometry is exported as a .iges file, since the grid generator needs this format.

\subsection{Grid generation}

For the grid generation, the unstructured hybrid grid generator CENTAUR [7] is used. For the 2D computing grid, different mesh elements including both triangles and quadrilaterals are used. The quadrilateral elements are generated in layers near the wall surfaces to capture the viscous boundary layer, while the triangular elements fill the remainder of the domain. The 3D grid generation uses triangular elements to generate the surface grid, prisms to capture the viscous boundary layer and tetrahedral cells to fill the remaining flow region. The whole grid generation process is based on three input files, for the surface mesh, for the size of the quadrilateral elements along the wall and for the triangular elements. They have to be adopted according to the desired grid resolution. Important factors, which influence the grid composition, are the Reynolds number, the chosen turbulence model and the near wall modeling. More information about this meshing tool can be found in [7]. To ensure grid independence, a large number of grid independence tests have been done in former studies [4].

\subsection{Flow field}

Since the flow in the cooling channel is turbulent, the turbulent stresses have to be considered by solving the conservation equations. This is done with the finite volume CFD code FLUENT [8]. For the turbulence modeling, a $k-\varepsilon$ turbulence model is used. Two different models are applied for the near wall treatment. Wall functions are applied for coarse grids, meaning that the $y^{+}$value for the first grid cell near the wall has to be around 30. For fine grids, a two-layer approach has been used. For the present case the momentum and turbulence equations are solved separately before the energy equations are solved. As thermal boundary condition, a constant wall temperature $T_{\mathrm{w}}$ is assumed. Since the flow is periodic, a mass flow rate depending on the Reynolds number is predefined. As convergence criterion, either a value for the residual or a maximum number of iterations is taken. Detailed information about the conservation equations used can be found in [8].

\section{RESULTS FOR THE SOGA}

In this section, results obtained with the SOGA are presented. Here a simplified 2D geometry of a rectangular cooling channel is used. The first point concerns the definition of an appropriate objective function. To show that the optimization results also give physically meaningful values, a comparison with experimental data is conducted. The last subsection concerns the optimal geometries which are obtained for the different objective functions. There are many parameters in a GA which can be changed or adapted depending on the problem under investigation. In a former study [11], 
the dependence of different parameters, such as the population size $N$ or binary coding, has been carried out. In the following section, the calculations are performed with a Reynolds number of $R e=u_{\mathrm{b}} D_{\mathrm{h}} / v=45,000$, a crossover rate of $p_{\mathrm{c}}=0.8$, a mutation rate of $p_{\mathrm{m}}=0.01$ and a binary coding of $6 \times 4$.

\subsection{Geometry}

The rib roughened cooling channel is simplified to a $2 \mathrm{D}$ periodic domain with ribs on the bottom and top walls. As design parameters, the heights $e_{1}, e_{2}$ and the widths $b_{1}, b_{2}$ of the ribs, the distance between the ribs $d$ and the inner pitch distance or length of the channel $p$, are varied. This results in six parameters to be optimized, while the height of the channel is taken as constant ( $H=$ constant). Figure 5 shows the geometry with the described parameters. The parameters to be optimized are considered in the following range:

$$
\begin{gathered}
0.05 \leq e_{1} / H \leq 0.3, \\
0.05 \leq e_{2} / H \leq 0.3, \\
0.5 \leq b_{1} / e_{1} \leq 2.0, \\
0.5 \leq b_{2} / e_{2} \leq 2.0, \\
5 \leq 2 p /\left(e_{1}+e_{2}\right) \leq 20, \\
0 \leq d / p \leq 0.5 .
\end{gathered}
$$

The domain boundaries for the different decision variables are given according to experience from former studies.

\subsection{Objective function}

Since the main optimization objective in a cooling channel is the enhancement of the heat transfer, first, an objective function which contains only the Nusselt number is taken:

$$
g_{1}\left(x_{i}\right)=N u / N u_{0} .
$$

The Nusselt number is a measure for the heat transfer rate

$$
N u=h D_{\mathrm{h}} / k
$$

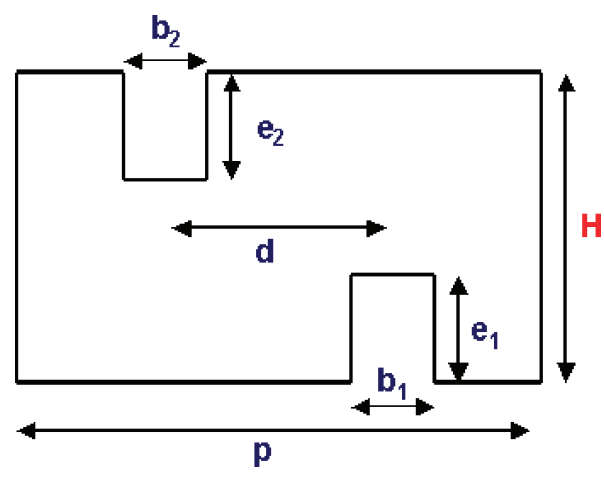

Figure 5: Periodic segment with definition of the decision variables. 
and is often normalized by the Nusselt number in a smooth duct. Here the Dittus-Boelter correlation has been used as a reference [12]:

$$
N u_{0}=0.023 \operatorname{Re}^{0.8} \operatorname{Pr}^{0.4}
$$

The second relationship for the objective function contains the Nusselt number $N u$ and the friction factor $f$, since the pressure loss also has an influence on the performance of a cooling channel and should be as low as possible. The fanning friction factor [12] is defined by

$$
f=\left(D_{\mathrm{h}} \Delta p\right) /\left(2 \rho u_{\mathrm{b}}^{2} p\right) .
$$

The following relation, which is originally derived from heat exchanger considerations, is often taken into account to benchmark different cooling channel designs and will be used in the present study as a second objective function:

$$
g_{2}\left(x_{i}\right)=\left(N u / N u_{0}\right) /\left(f / f_{0}\right)^{1 / 3} .
$$

The friction factor, appearing in the denominator of eqn (11), is normalized by the correlation for the friction factor in a smooth duct:

$$
f_{0}=0.046 R e^{-0.2}
$$

\subsection{Validation of results}

To validate the numerical calculations, the experimental data of Han [13] have been taken into consideration. Han investigated the local heat transfer coefficients in rectangular channels with two opposite ribbed walls. The channel width-to-height ratio for one setup was $W / H=4$, which can be compared approximately to the flow in a $2 \mathrm{D}$ channel. The rib height-to-hydraulic diameter ratio was $e / D_{\mathrm{h}}=0.078$ and the rib angle of attack was kept at $90^{\circ}$. The flow was investigated at $R e=30,000$. Table 1 compares the Nusselt number and friction factor obtained by 2D numerical calculations with the experimental results of Han. One can see that the difference between numerical and experimental results is below $15 \%$, which is acceptable due to the $2 \mathrm{D}$ simplification and the average measurement uncertainties.

\subsection{Optimal geometry}

4.4.1 Objective function $g_{1}=N u / N u_{0}$

For the first objective function, which depends only on the Nusselt number, the optimal geometries can be generalized by the following characteristics: small high ribs, approaching the maximum value of the rib height and the minimum value for the rib width $b$. The inner pitch distance

Table 1: Comparison between numerical results and experimental data of Han [13].

\begin{tabular}{lccc}
\hline & Numerical & Experimental & $\begin{array}{c}\text { Relative } \\
\text { difference (\%) }\end{array}$ \\
\hline $\mathrm{Nu} / \mathrm{Nu}_{0}$ & 2.18 & 2.55 & 14.5 \\
$\mathrm{f} / \mathrm{f}_{0}$ & 13.9 & 15.5 & 10.3 \\
\hline
\end{tabular}


$2 p /\left(e_{1}+e_{2}\right)$ is small and the distance between the ribs takes values between 0.1 and 0.4. Data describing the nearly optimal geometries can be found in Table 2. A typical geometry can be seen in Fig. 6a.

4.4.2 Objective function $g_{2}=\left(N u / N u_{0}\right) /\left(f / f_{0}\right)^{1 / 3}$

For the second objective function, taking the friction factor and the Nusselt number into account, totally different rib geometries are obtained. Typical geometrical parameters are presented in Table 3 and a typical geometry can be seen in Fig. 6b. The geometries can be summarized by the following points: flat ribs, which are for the height near the lower boundaries of the decision variables. A typical width for the ribs cannot be given; the ribs on the bottom and top walls do not necessarily have the same shape as can be seen in Fig. 6 b. The channels have a very high inner pitch distance reaching mostly the maximum value of $2 p /\left(e_{1}+e_{2}\right)=20$. The absolute length does not change strongly compared to the length of the channel obtained with the objective function $g_{1}$ due to the lower rib height. The distance between the ribs takes values between staggered ribs $d / p=0.5$ and smaller distances $d / p=0.333$.

Table 2: Three nearly optimal geometries obtained at different optimization runs for the first objective function $g_{1}$.

\begin{tabular}{ccccccc}
\hline$e_{1} / H$ & $e_{2} / H$ & $b_{1} / H$ & $b_{2} / H$ & $2 p /\left(e_{1}+e_{2}\right)$ & $d / p$ & $g_{1}$ \\
\hline 0.3 & 0.3 & 0.5 & 0.5 & 5 & 0.4 & 4.260 \\
0.3 & 0.3 & 0.6 & 0.5 & 5 & 0.1 & 4.151 \\
0.3 & 0.3 & 0.5 & 0.5 & 5 & 0.4333 & 4.266 \\
\hline
\end{tabular}

(a)

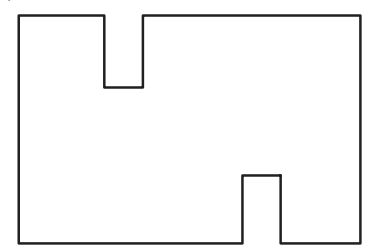

(b)

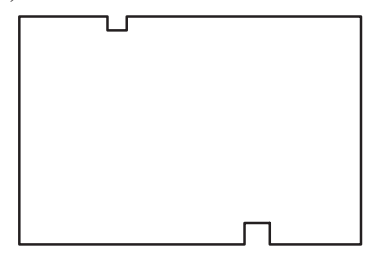

Figure 6: Optimal geometry obtained with two objective functions: (a) an optimum geometry for $g_{1}=N u / N u_{0}$ and (b) an optimum geometry for $g_{2}=\left(N u / N u_{0}\right) /\left(f / f_{0}\right)^{1 / 3}$.

Table 3: Three nearly optimal geometries obtained at different optimization runs for the second objective function $g_{2}$.

\begin{tabular}{lcccccc}
\hline$e_{1} / H$ & $e_{2} / H$ & $b_{1} / H$ & $b_{2} / H$ & $2 p /\left(e_{1}+e_{2}\right)$ & $d / p$ & $g_{2}$ \\
\hline 0.0500 & 0.0833 & 1.9 & 0.7 & 19 & 0.47 & 0.859 \\
0.0667 & 0.0833 & 1.0 & 1.2 & 20 & 0.4 & 0.862 \\
0.0500 & 0.0667 & 1.4 & 0.7 & 20 & 0.3 & 0.855 \\
\hline
\end{tabular}




\section{RESULTS FOR THE MOGA}

As shown in the previous section, the optimal geometry is strongly dependent on the chosen objective function. To fulfill two conflicting objectives at the same time, a multi-objective optimization is more practical. For internal cooling channels, the main optimization targets are the maximization of the Nusselt number ratio $N u / N u_{0}$ and a reduction of the friction factor ratio $f / f_{0}$; thus, these normalized quantities are taken as objective functions:

$$
\begin{gathered}
\max g_{1}=N u / N u_{0}, \\
\min g_{2}=f / f_{0} .
\end{gathered}
$$

The two main optimization goals of a MOGA are to find a set of solutions as close as possible to the POF and place the individual along this front as diverse as possible to display the devolution as clearly as possible.

\subsection{The 2D problem}

The optimization was carried out with a population size of $N=20$, a crossover probability of $p_{\mathrm{c}}=0.8$ and a binary coding of $6 \times 4$ at two Reynolds numbers of $R e=50,000$ and $R e=100,000$. Figure 7 shows the POF in the normalized magnitudes of $N u / N u_{0}$ and $f / f_{0}$. For both Reynolds numbers the same curve is obtained; there is only a small shift to higher values for the higher Reynolds number. Table 4 shows the geometry definitions of the individuals for $R e=100,000$. From the geometrical data, one can observe that the optimal geometries in the upper part of the POF have similar geometry characteristics as the optimal geometries of the first objective function in the SOGA. The geometries along the lower part of the POF cannot be generally characterized; they have smaller ribs and a larger inner pitch distance but the shape is strongly changing. The geometry with the lowest pressure loss has similar decision variables as the optimal geometry of the second objective function in the SOGA.

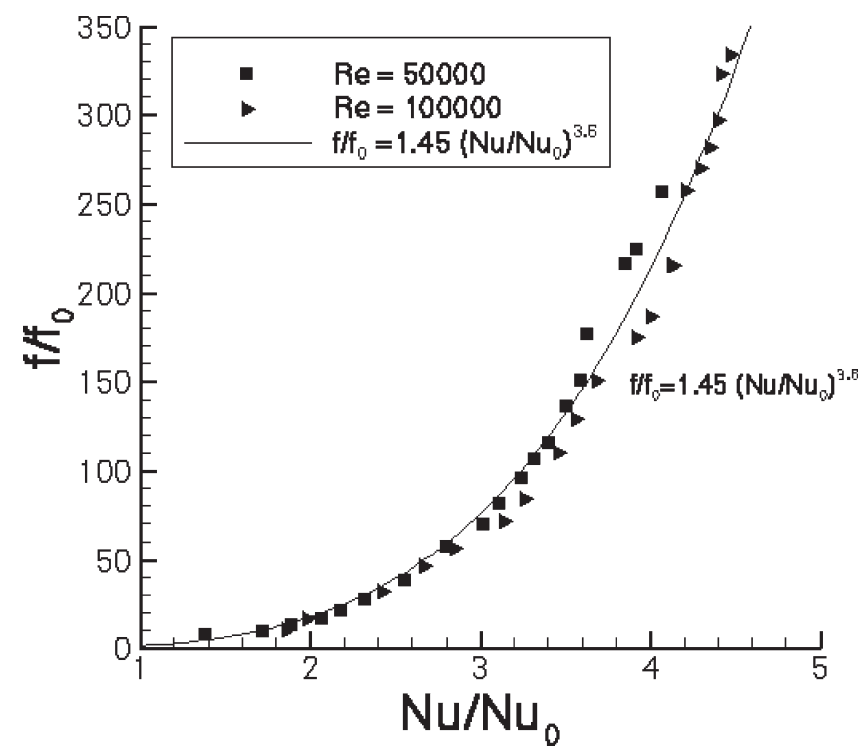

Figure 7: POF for normalized magnitudes $N u / N u_{0}$ and $f / f_{0}$. 
Table 4: Geometry parameters for the POF at $R e=100,000$.

\begin{tabular}{rrlcccrr}
\hline$e_{1} / H$ & $e_{2} / H$ & $b_{1} / H$ & $b_{2} / H$ & $2 p /\left(e_{1}+e_{2}\right)$ & $D / p$ & $N u$ & $\Delta p$ \\
\hline 0.283 & 0.283 & 0.5 & 0.5 & 5 & 0.500 & 915 & $-1,003$ \\
0.283 & 0.283 & 0.6 & 0.5 & 5 & 0.500 & 903 & -971 \\
0.283 & 0.300 & 0.5 & 0.5 & 6 & 0.333 & 899 & -892 \\
0.283 & 0.300 & 0.5 & 0.5 & 6 & 0.500 & 890 & -846 \\
0.283 & 0.300 & 0.7 & 0.5 & 6 & 0.466 & 878 & -811 \\
0.283 & 0.283 & 0.7 & 0.5 & 6 & 0.466 & 860 & -773 \\
0.283 & 0.283 & 0.5 & 0.5 & 7 & 0.500 & 846 & -647 \\
0.283 & 0.283 & 0.7 & 0.5 & 7 & 0.500 & 822 & -616 \\
0.283 & 0.283 & 0.5 & 0.5 & 8 & 0.466 & 802 & -525 \\
0.283 & 0.283 & 1.0 & 0.5 & 8 & 0.466 & 768 & -478 \\
0.283 & 0.283 & 1.4 & 0.5 & 8 & 0.500 & 753 & -452 \\
0.183 & 0.283 & 0.6 & 0.5 & 8 & 0.466 & 728 & -387 \\
0.283 & 0.150 & 0.6 & 0.5 & 8 & 0.466 & 697 & -323 \\
0.283 & 0.283 & 0.5 & 0.5 & 13 & 0.500 & 668 & -253 \\
0.150 & 0.150 & 0.6 & 0.5 & 7 & 0.500 & 642 & -214 \\
0.166 & 0.283 & 0.5 & 0.5 & 13 & 0.500 & 610 & -189 \\
0.150 & 0.283 & 0.5 & 0.9 & 13 & 0.500 & 583 & -168 \\
0.283 & 0.283 & 0.6 & 1.8 & 20 & 0.366 & 511 & -119 \\
0.150 & 0.083 & 0.083 & 1.8 & 12 & 0.466 & 468 & -75 \\
0.050 & 0.066 & 1.7 & 1.8 & 20 & 0.366 & 381 & -31 \\
\hline
\end{tabular}

Generally, it can be seen that the whole spectrum of possible values is obtained by the six decision variables. To find a curve which describes the position of the optimal geometries along the POF, a least-squares algorithm is applied leading to the following function:

$$
f / f_{0}=1.45\left(\mathrm{Nu} / \mathrm{Nu} u_{0}\right)^{3.6} .
$$

Equation (13) is of high importance for the industrial layout of a cooling channel because a designer knows that he has no optimal geometry if his channel is found on the left side of the graph obtained by the given equation. Depending on the given problem, one can decide to have a higher heat transfer taking the same pressure loss into account by simply moving to the right until one reaches the curve or to reduce the pressure loss and keep the given heat transfer rate by moving down along a straight line until the optimal curve is reached.

\subsection{The 3D problem}

For the 3D optimization problem, two different cooling channel geometries have been investigated: a square channel with ribs on the top and bottom walls and a channel typically for a leading edge region of a gas turbine blade with a nearly triangular cross section.

To reduce the computational time because of the large grid sizes (up to 400,000 cells), the calculations are performed in parallel. This results in 20 individuals per generation in a 20 times reduced calculation time. Another possibility to speed up the optimization algorithm is to use for the first generations a simple turbulence description, which allows a coarser grid resolution (i.e. $k-\varepsilon$ model 
with wall functions), followed by a model with a higher grid resolution for the final results. In the given example, the difference in the computational costs between the classical 2D and 3D square channel is approximately 20 times.

5.2.1 Channel with square cross section

For the square cooling channel, six decision variables are chosen: the normalized rib height on the bottom wall $e_{1} / D_{\mathrm{h}}$ and on the top wall $e_{2} / D_{\mathrm{h}}$; the rib width, defined by one value giving the relation $b / e_{1}$ or $b / e_{2}$ for both walls; the inner pitch distance $p$ given over the average value for the rib height; the distance between the ribs $d$ and the rib angle $\alpha$. The decisions are given in the following range:

$$
\begin{gathered}
0.05 \leq e_{1} / D_{\mathrm{h}} \leq 0.25, \\
0.05 \leq e_{2} / D_{\mathrm{h}} \leq 0.25, \\
0.5 \leq b_{1} / e_{1 / 2} \leq 1.0, \\
5 \leq 2 p /\left(e_{1}+e_{2}\right) \leq 20, \\
0 \leq d / p \leq 0.5, \\
30^{\circ} \leq \alpha \leq 90^{\circ} .
\end{gathered}
$$

The optimization was performed with the $k-\varepsilon$ turbulence model. For the first 20 generations, the near wall region was modeled with the wall function approach, which results in less grid cells. For the remaining generations, the two-layer approach was applied. More information concerning the grid resolution can be found in [14].

In Fig. 8, the POF for the square cooling channel is shown for the normalized friction factor $f / f_{0}$ and the normalized Nusselt number $N u / N u_{0}$. The geometries in the upper part of the POF have high

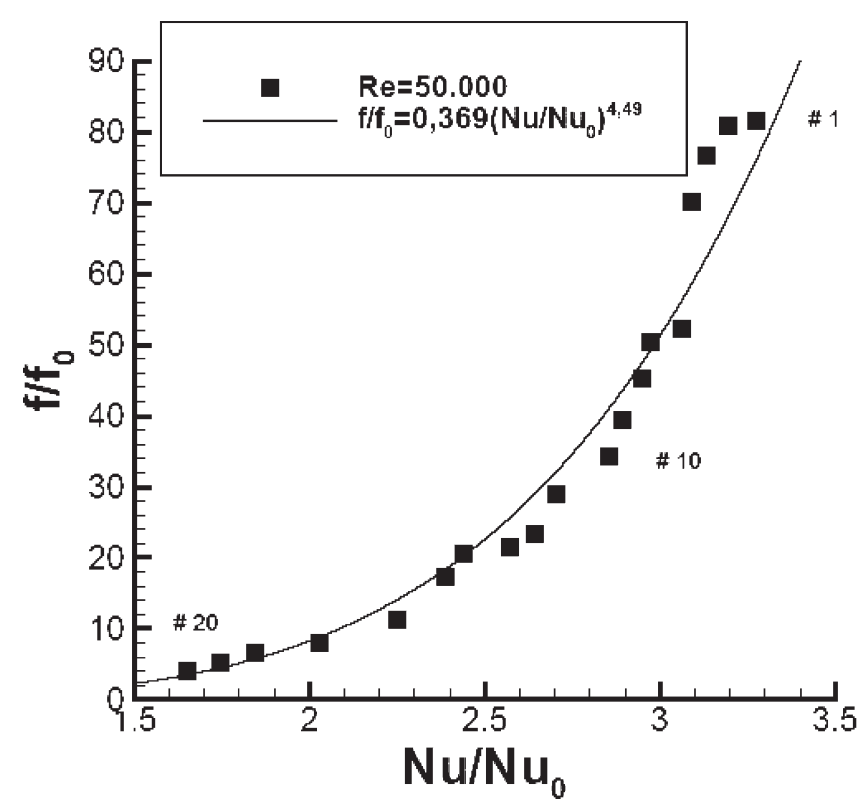

Figure 8: POF of the square cooling channel at $R e=50,000$. 
rib heights $e_{1} / D_{\mathrm{h}}, e_{2} / D_{\mathrm{h}}$, small inner pitch distances $2 p /\left(e_{1}+e_{2}\right)$ and small rib angles $\alpha$. In the lower part of the POF, one finds small rib height, large inner pitch distances and rib angles around $\alpha=30^{\circ}$.

The rib width $b$ and the distance between the ribs $d$ varies along the POF. Examples of the optimal geometries for this case are shown in Fig. 9.

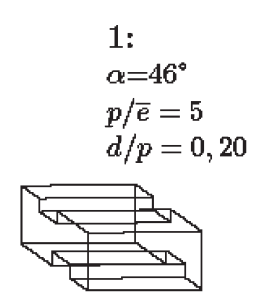

5:

$\alpha=46^{\circ}$

$p / \bar{e}=5$

$d / p=0,07$

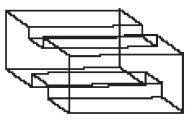

9:

$\alpha=46^{\circ}$

$p / \bar{e}=5$

$d / p=0,07$

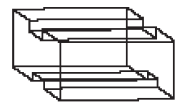

13:

$\alpha=46^{\circ}$

$p / \bar{e}=5$

$d / p=0,07$

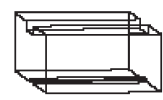

17:

$\alpha=30^{\circ}$

$p / \bar{e}=18$

$d / p=0,27$
2:

$\alpha=46^{\circ}$

$p / \bar{e}=5$

$d / p=0,06$

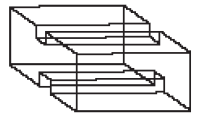

$6:$

$\alpha=50^{\circ}$

$p / \bar{e}=5$

$d / p=0,07$

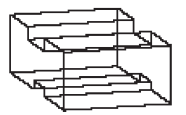

10:

$\alpha=50^{\circ}$

$p / \bar{e}=5$

$d / p=0,20$

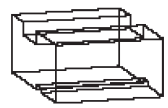

14:

$\alpha=46^{\circ}$

$p / \bar{e}=5$

$d / p=0,27$

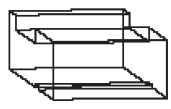

18:

$\alpha=30^{\circ}$

$p / \bar{e}=14$

$d / p=0,00$
3:

$\alpha=46^{\circ}$

$p / \bar{e}=5$

$d / p=0,20$

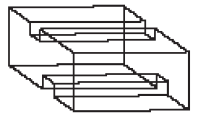

7:

$\alpha=46^{\circ}$

$p / \bar{e}=5$

$d / p=0,07$

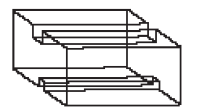

11:

$\alpha=46^{\circ}$

$p / \bar{e}=6$

$d / p=0,20$

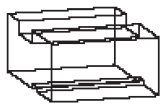

15:

$\alpha=50^{\circ}$

$p / \bar{e}=5$

$d / p=0,07$

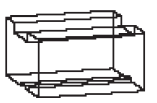

19:

$\alpha=30^{\circ}$

$p / \bar{e}=18$

$d / p=0,27$
4:

$\alpha=46^{\circ}$

$p / \bar{e}=5$

$d / p=0,06$

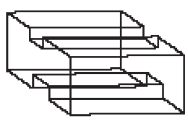

8:

$\alpha=46^{\circ}$

$p / \bar{e}=5$

$d / p=0,07$

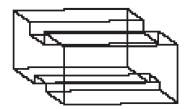

12:

$\alpha=46^{\circ}$

$p / \bar{e}=6$

$d / p=0,33$

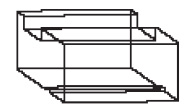

16:

$\alpha=50^{\circ}$

$p / \bar{e}=5$

$d / p=0,07$

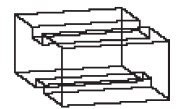

20:

$\alpha=30^{\circ}$

$p / \bar{e}=18$

$d / p=0,33$
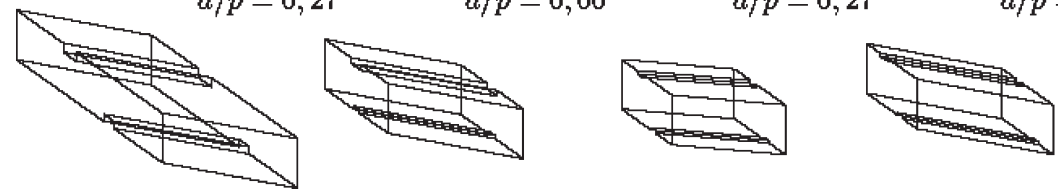

Figure 9: Different optimal rib configurations in the square cooling channel. 
The function describing the shape of the POF is given by

$$
f / f_{0}=0.367\left(N u / N u_{0}\right)^{4.49} .
$$

\subsubsection{Leading edge channel}

The leading edge channel has a shape similar to an acute-angled triangle with one rounded corner. This shape fits into the leading edge region of a turbine blade. Ribs are mounted on the pressure and suction side to enhance the heat transfer. The geometry is depicted in Fig. 10.

In contrast to the square cooling channels with ribs having a constant cross section, the ribs of the leading edge channel are reduced in height according to the free flow section. The two reference values are the maximum rib height $e_{\max }$ at the back wall and the minimal rib height $e_{\min }$ at the boundary to the rounded corner. Between these values, the rib height varies linearly. For the optimization process, the two reference values are related to constant sizes, which are given through the channel geometry, the length $H_{\max }$ of the smooth and even wall and the length $H_{\min }$, which is the cross section at the beginning of the arc.

The following decision variables have been defined for the optimization process: the normalized maximal rib height $e_{\max } / H_{\max }$ and the normalized minimal rib height $e_{\min } / H_{\min }$ as described earlier. The normalized rib height $2 b /\left(e_{\max }+e_{\min }\right)$, the inner pitch distance $p$ given for the average value of the rib height, the distance between the ribs $d$ and the rib angle $\alpha$. Here no difference will be made for the ribs on the suction and pressure side. The decisions are made in the following range:

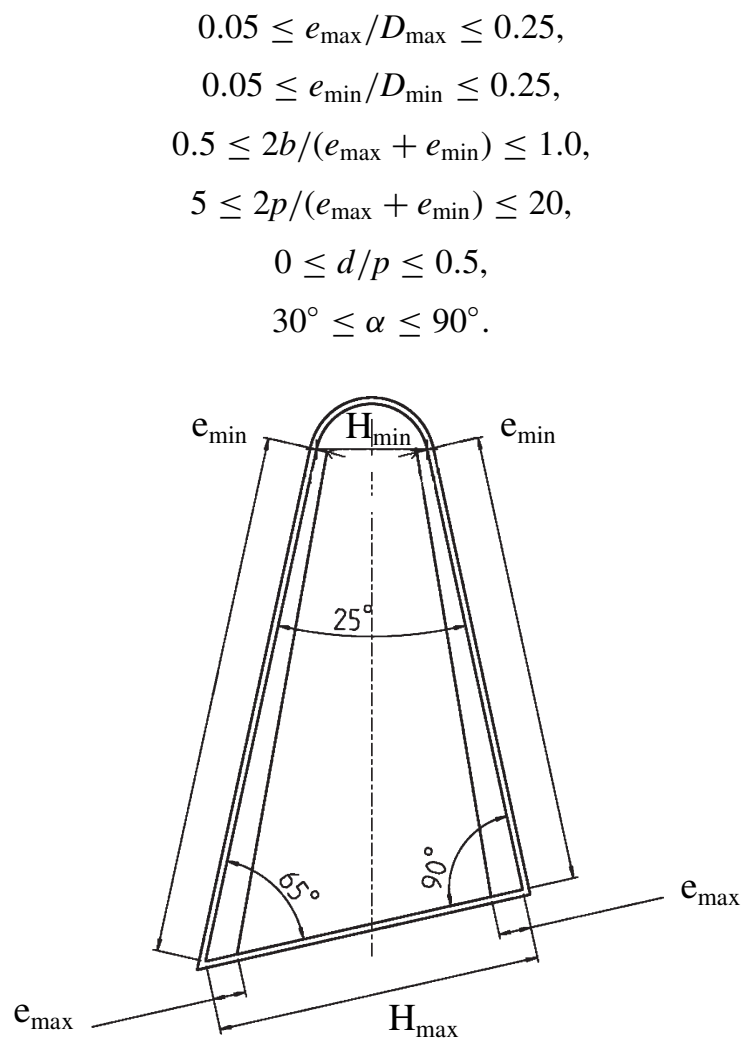

Figure 10: Geometry for the leading edge channel. 


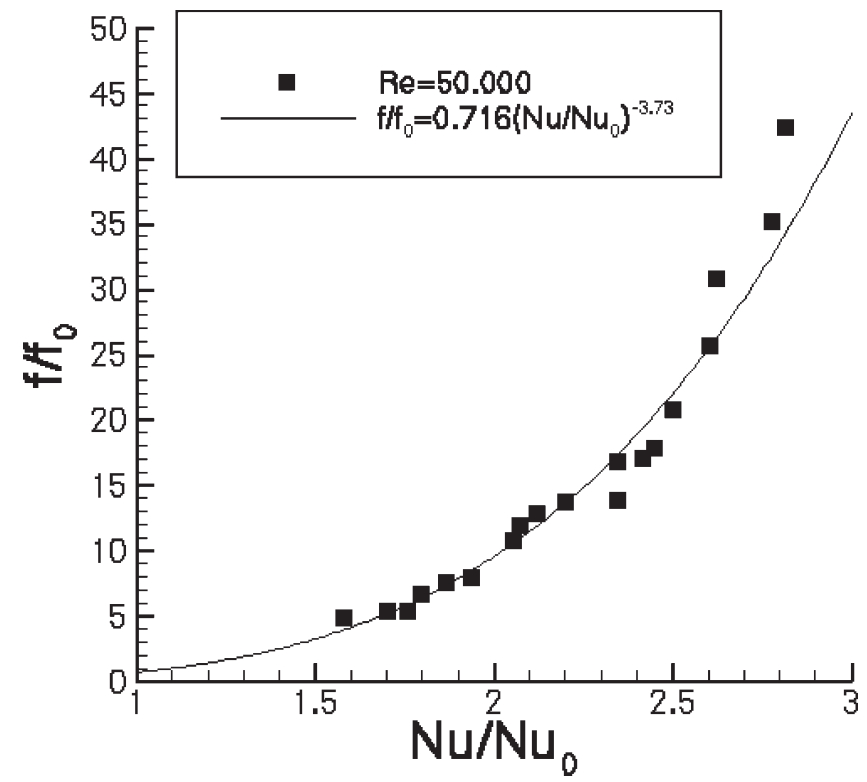

Figure 11: POF for the leading edge channel at $R e=50,000$.

During the optimization process, a $k-\varepsilon$ model with a two-layer approach for the near wall region is applied. For the grid resolution, known parameters were taken from a former study. Details can be found in [14]; the average grid size was around 400,000 cells.

For the MOGA, similar parameters are taken as for the $2 \mathrm{D}$ optimization. The whole optimization was done for a Reynolds number of 50,000. In Fig. 11, the POF for the leading edge channel is shown. For most geometries, the value $e_{\min }$ has very low values, which can be explained by the minor blocking of the flow in the corner region. For the maximal rib height $e_{\max }$ all values will be passed through; the higher values can be found in the upper part of the POF with high Nusselt numbers and large pressure losses, whereas the low values for $e_{\max }$ are in the lower part of the POF. For the other design variable, no general trend can be found. Only for rib angle, it can be observed that small angles are preferred for most optimal geometries. Examples of the optimal geometries for this case are shown in Fig. 12.

Again, a function describing the shape of the POF can be defined by

$$
f / f_{0}=0.716\left(N u / N u_{0}\right)^{3.73} .
$$

\section{CONCLUSIONS}

The present study demonstrates how GAs can be used for the optimization of the rib structure inside cooling channels. The main aim of the SOGA is to maximize or minimize a single objective function; the MOGA is able to optimize two or more functions. In the SOGA, two different objective functions $g_{1}=N u / N u_{0}$ and $g_{2}=\left(N u / N u_{0}\right) /\left(f / f_{0}\right)^{1 / 3}$ have been used for the optimization. The nearly optimal channels have different shapes depending on the chosen objective function.

The MOGA seems to be better suited for the optimization with two objectives because the whole POF can be calculated. A function which describes the devolution of the POF for a square cooling channel and a leading edge channel is determined. This function can be used to evaluate a geometry 


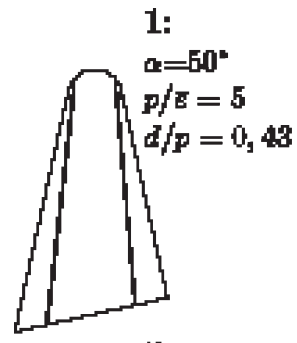

5:

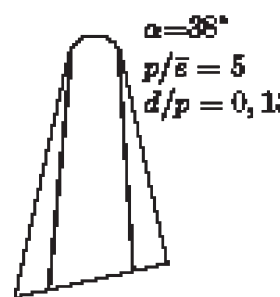

9:

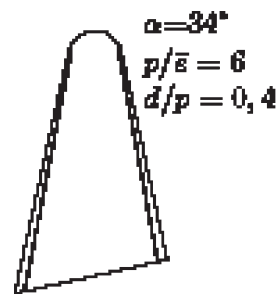

18:

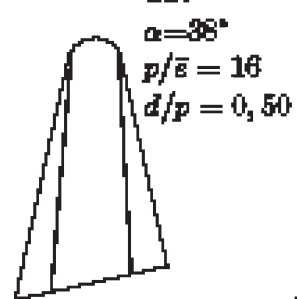

17:

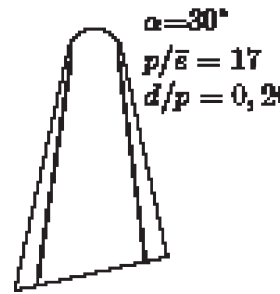

2:

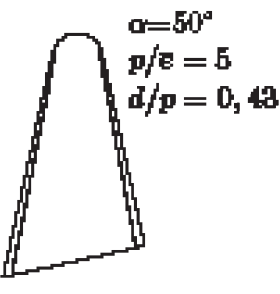

6:

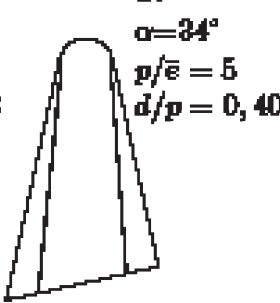

10:

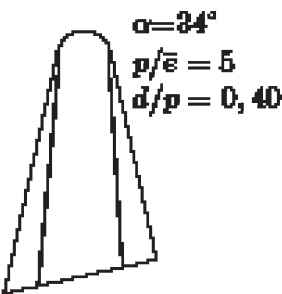

14:

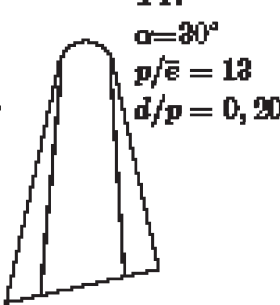

18:

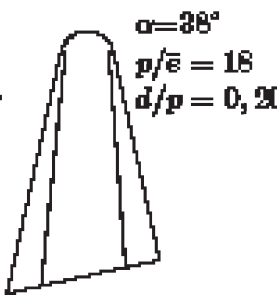

3:

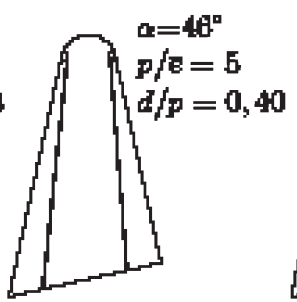

7:

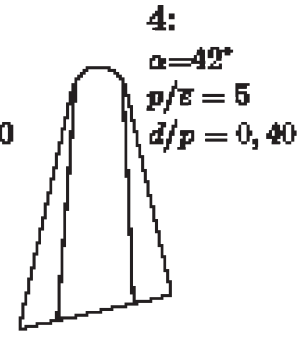

8:

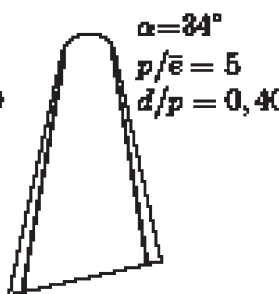

11:

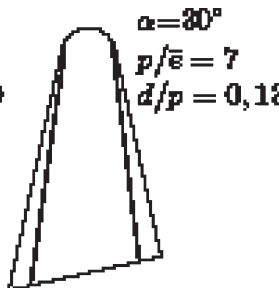

15:

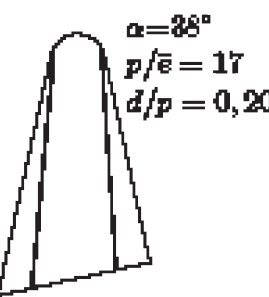

19:

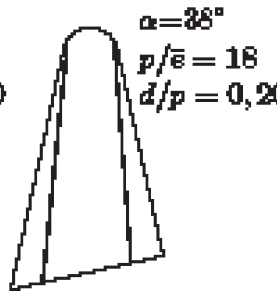

20:

$a=00^{\circ}$

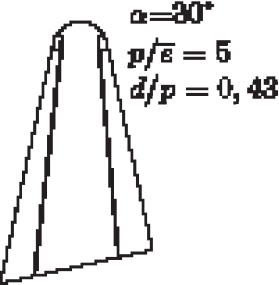

16:

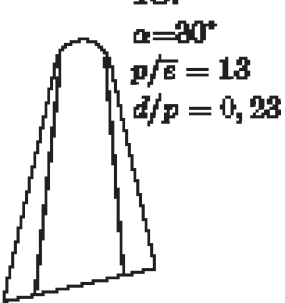

12:

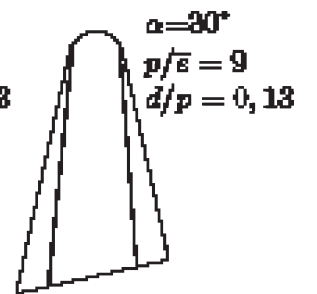

$=34^{*}$

$p / \sqrt{e}=6$

$d / p=0,40$

Figure 12: Different optimal configurations for the leading edge channel.

concerning their quality. For geometries which are apart from this curve, an enhancement of the Nusselt number or a reduction of the friction factor can be obtained. The overall performance of the GAs shows that it is a good method for the optimization of the rib structure in internal cooling channels. 
$b \quad$ rib width $(\mathrm{m})$

NOMENCLATURE

$D_{\mathrm{h}} \quad$ hydraulic diameter $(\mathrm{m})$

$d \quad$ distance between two ribs (m)

$e \quad$ rib height

$f \quad$ friction factor

$f_{0} \quad$ friction factor for a smooth duct

$g_{1 / 2} \quad$ objective function

$H \quad$ channel height $(\mathrm{m})$

$h$ heat transfer coefficient $\left(\mathrm{W} / \mathrm{m}^{2} \mathrm{~K}\right)$

$k$ thermal conductivity $(\mathrm{W} / \mathrm{mK})$

$N \quad$ population size

$\mathrm{Nu} \quad$ Nusselt number

$N u_{0} \quad$ Nusselt number for a smooth duct

Pr Prandl number

$p \quad$ inner pitch or channel length (m)

$p_{\mathrm{c}} \quad$ crossover probability

$p_{\mathrm{m}} \quad$ mutation probability

Re Reynolds number

$u_{\mathrm{b}} \quad$ channel bulk velocity $(\mathrm{m} / \mathrm{s})$

$W \quad$ channel weight (m)

()$_{2}$ number in binary system

( ) 10 number in decimal system

$\Delta p \quad$ pressure loss $(\mathrm{Pa})$

$\rho \quad$ density $\left(\mathrm{kg} / \mathrm{m}^{3}\right)$

$v \quad$ kinematic viscosity $\left(\mathrm{m}^{2} / \mathrm{s}\right)$

\section{ACKNOWLEDGMENT}

The authors acknowledge the helpful discussions with Dr M. Schnieder from ALSTOM Power Baden/Switzerland concerning the calculations presented in this paper.

\section{REFERENCES}

[1] Han, J.C. \& Dutta, S., Internal convection heat transfer and cooling - an experimental approach; fundamental heat transfer in stationary ribbed channels. VKI Lecture Series, 1995-05, Heat Transfer and Cooling in Gas Turbines, 1995.

[2] Han, J.C., Dutta, S. \& Ekkad, S.V., Gas Turbine Heat Transfer and Cooling Technology, Taylor \& Francis: New York, 2000.

[3] Weigand, B., Semmler, K. \& von Wolfersdorf, J., Heat transfer technology for internal passages of air-cooled blades for heavy-duty gas turbines. Annals of the New York Academy of Science, 934(1), pp. 179-193, 2001.

[4] Haasenritter, A., Amro, M. \& Weigand, B., An experimental and numerical study of the heat transfer performance of sharp-edged and rounded ribs in square ducts. The 9th International Symposium on Transport Phenomena and Dynamics of Rotating Machinery, Honolulu, 2002.

[5] Hermanson, K., Parneix, S., von Wolfersdorf, J. \& Semmler, K., Prediction of pressure loss and heat transfer in internal cooling passages. Int. Symposium, Heat Transfer in Gas turbine Systems, August 2000. 
[6] Koehler, P., Hoffmann, R. \& Koehler, M., Pro/Engineer-Praktikum, Vieweg, Studium Technik, 2000.

[7] Kallinderis, Y., Khawaja, A. \& McMorris, H., Hybrid prismatic/tetrahedral grid generation for flows around complex geometries. AIAA Journal, 34, pp. 291-298, February 1996.

[8] FLUENT Manual, FLUENT5 User's Guide, Vol. 1-5, FLUENT Inc.: New Hampshire, July 1998.

[9] Deb, K., Optimization for Engineering Design, Prentice-Hall: New Delhi, 1998.

[10] Deb, K., Multi-Objective Optimization Using Evolutionary Algorithms, John Wiley \& Sons Ltd.: Chichester, 2002.

[11] Haasenritter, A. \& Weigand, B., Optimization of the rib structure inside a 2D cooling channel. Proceedings of ASME Turbo Expo 2004, Vienna, Austria, June 14-17, 2004.

[12] Kays, W., Crawford, M. \& Weigand, B., Convective Heat and Mass Transfer, McGraw Hill: New York, 2004.

[13] Han, J.C., Heat transfer and friction characteristics in rectangular channels with rib turbulators. Journal of Heat Transfer, 110, May 1988.

[14] Haasenritter, A., Numerische Untersuchung und Optimierung von berippten Kühlkanalgeometrien für Gasturbinenschaufeln mit Genetischen Algorithmen, Dissertation, Universität Stuttgart, 2005. 